\title{
A case study of a long-term glioblastoma survivor with unmethylated MGMT and hypermutated genotype
}

\author{
Toni Rose Jue, ${ }^{1,5}$ Lauren R. Olafson, ${ }^{1,5}$ Anna H. Siddell, ${ }^{1}$ Robert W. Rapkins, ${ }^{1}$ \\ Benedict Ng, ${ }^{1}$ Julia X.M. Yin, ${ }^{1}$ Victor M. Lu, ${ }^{1}$ Sylvia A. Chung, ${ }^{1}$ Shane P. Whittaker, ${ }^{1}$ \\ Matthew Davies, ${ }^{1}$ Jacob M. Fairhall, ${ }^{2,3}$ Elizabeth J. Hovey, ${ }^{3,4}$ \\ and Kerrie L. McDonald ${ }^{1}$

\begin{abstract}
${ }^{1}$ Cure Brain Cancer Biomarkers and Translational Research Group, Prince of Wales Clinical School, University of New South Wales, Sydney, New South Wales 2052, Australia; ${ }^{2}$ Neurospine Clinic, Prince of Wales Hospital, Randwick, New South Wales 2031, Australia; ${ }^{3}$ University of New South Wales, Sydney, New South Wales 2031, Australia; ${ }^{4}$ Department of Medical Oncology, Nelune Comprehensive Cancer Centre, Prince of Wales,
\end{abstract} \\ Hospital, Randwick, New South Wales 2031, Australia
}

Corresponding author: k.mcdonald@unsw.edu.au

(c) 2019 Jue et al. This article is distributed under the terms of the Creative Commons Attribution-NonCommercial License, which permits reuse and redistribution, except for commercial purposes, provided that the original author and source are credited.

Ontology terms: glioblastoma; glioma

Published by Cold Spring Harbor Laboratory Press

doi:10.1101/mcs.a003251
Abstract Effective treatments that extend survival of malignant brain tumor glioblastoma (GBM) have not changed in more than a decade; however, there exists a minority patient group $(<5 \%)$ whose survival is longer than $3 \mathrm{yr}$. We herein present a case report of a long-term surviving 51-yr-old female diagnosed with a MGMT unmethylated GBM. The patient was progression-free for $23 \mathrm{mo}$. Fresh primary and recurrent tumor samples were collected and processed for patient-derived model development. Whole-genome sequencing (WGS) was performed concurrently with additional standard of care diagnostics. WGS revealed a hypermutated genotype in the germline tissue and in both the primary and recurrent tumor samples. Specific to the matched tumors, an average of 30 cancer driver genes were mutated. Noteworthy was the identification of a nonsynonymous mutation in the POLE gene. As a possible instigator of the hypermutational genotype observed in the tumors, we identified nonsynonymous germline mutations within the mismatch repair genes, MLH1 and PMS2. Mutations within these genes are often indicative of the pan-cancer phenotype known as Lynch syndrome; however, their pathogenicity remains unreported. We performed a drug screen of 165 compounds, which identified one compound, YM155, an experimental survivin inhibitor, that showed effectivity to the patient-derived cell lines of both tumors. Treatment selection based on a patient's genome to individualize treatment for GBM patients could potentially be useful in the clinic. This is a promising avenue for further translational research, with larger databases and integrated platforms to increase the efficiency of analyzing and interpreting the individual genomic data of GBM.

[Supplemental material is available for this article.]

\section{INTRODUCTION}

Glioblastoma (GBM) remains one of the biggest therapeutic challenges in neuro-oncology. The median survival of people diagnosed with GBM is <15 mo (Minniti et al. 2009; Stupp

\footnotetext{
${ }^{5}$ These authors contributed equally to this work.
} 
et al. 2009). Very little improvement in survival times has been measured since temozolomide (TMZ) was introduced to the standard therapy regimen, back in 2005. Inherent intraand intertumoral heterogeneity has made treatment challenging (Jue and McDonald 2016). Selection of better therapies based on the genomics of each particular tumor is needed. Promising results from other cancer types have shown longer progression-free survival in the use of molecular profiling for target selection to determine possible treatment options (Von Hoff et al. 2010).

Herein, we present a case report of a patient diagnosed with primary GBM who had a recurrence $2 \mathrm{yr}$ after the initial diagnosis. Both primary and recurrent tumors were sent for standard of care diagnostics and biomarker screening. Both tumor samples taken from the time of resection were also processed for whole-genome sequencing (WGS). At the time of recurrence, a network analysis and drug-panel screening were performed on the recurrent tumor sample, as well as on the primary tumor sample for a retrospective comparison. This was performed to explore if an individualized treatment approach can be applied in the clinical management of patients diagnosed with GBM.

\section{RESULTS}

\section{Case Presentation}

A 51-yr-old female patient presented (April 2014) with a history of headaches, nausea, and alexia. Her previous medical history included a uterine leiomyoma (2010), paroxysmal atrial fibrillation, reflux esophagitis, and chronic anxiety. Magnetic resonance imaging (MRI) revealed a mass with hyperintense signal observed on the left temporoparietal lobe of the brain (Fig. 1A). The patient had no previous history of brain tumor malignancy. Maximal surgical resection with 5-aminolevulinic acid (5-ALA) infusion was performed. Histopathology confirmed GBM, IDH wild-type, O-6-methylguanine-DNA methyltransferase (MGMT) unmethylated, preserved nuclear ATRX staining, and negative EGFR immunohistochemistry (IHC) staining. A freshly resected tumor sample was processed for WGS; however, this was performed for research purposes rather than guiding management. The patient was treated with the standard EORTC protocol consisting of concurrent chemoradiation with continuous TMZ followed by 6 mo of adjuvant TMZ (5-d schedule). Despite being MGMT unmethylated (which is typically associated with a poorer response to TMZ), the patient's disease remained stable for 23 mo. There was an increase in T2/FLAIR changes on MRI (March 2016), and she was rechallenged with oral TMZ. Subsequent MRI scan (May 2016) demonstrated enlarging T2/FLAIR lesions, and TMZ was ceased. This prompted a new treatment approach consisting of pembrolizumab, a PD-1 inhibitor, given as a monotherapy. Pembrolizumab treatment was given over a course of four cycles, before an MRI scan revealed a new left frontal mass (Fig. 1B). The patient again underwent repeat tumor resection (September 2016), and histopathology was subsequently confirmed as recurrent GBM, with a new appearance of strong EGFR immunohistochemistry positivity (MGMT remained unmethylated). Part of the recurrent tumor sample was again processed for WGS. The patient was reirradiated with radiation directed at the bed of the left frontal lesion (November 2016). Concurrently the patient was also treated with palliative bevacizumab therapy, a monoclonal antibody to inhibit VEGF. Several lesions distant from the original tumor bed were noted on MRI scanning. The patient was then treated with ongoing bevacizumab and ABT-414, a novel EGFR inhibitor. TMZ was added to the ABT-414 for one cycle but the patient experienced marked myelosuppression so ABT-414 was continued as a monotherapy. MRI demonstrated further progression. Unfortunately, the patient continued to deteriorate, becoming bedbound, and in the end was sent for palliative care at home. The patient passed away 42 mo (October 2017) after her initial diagnosis. 

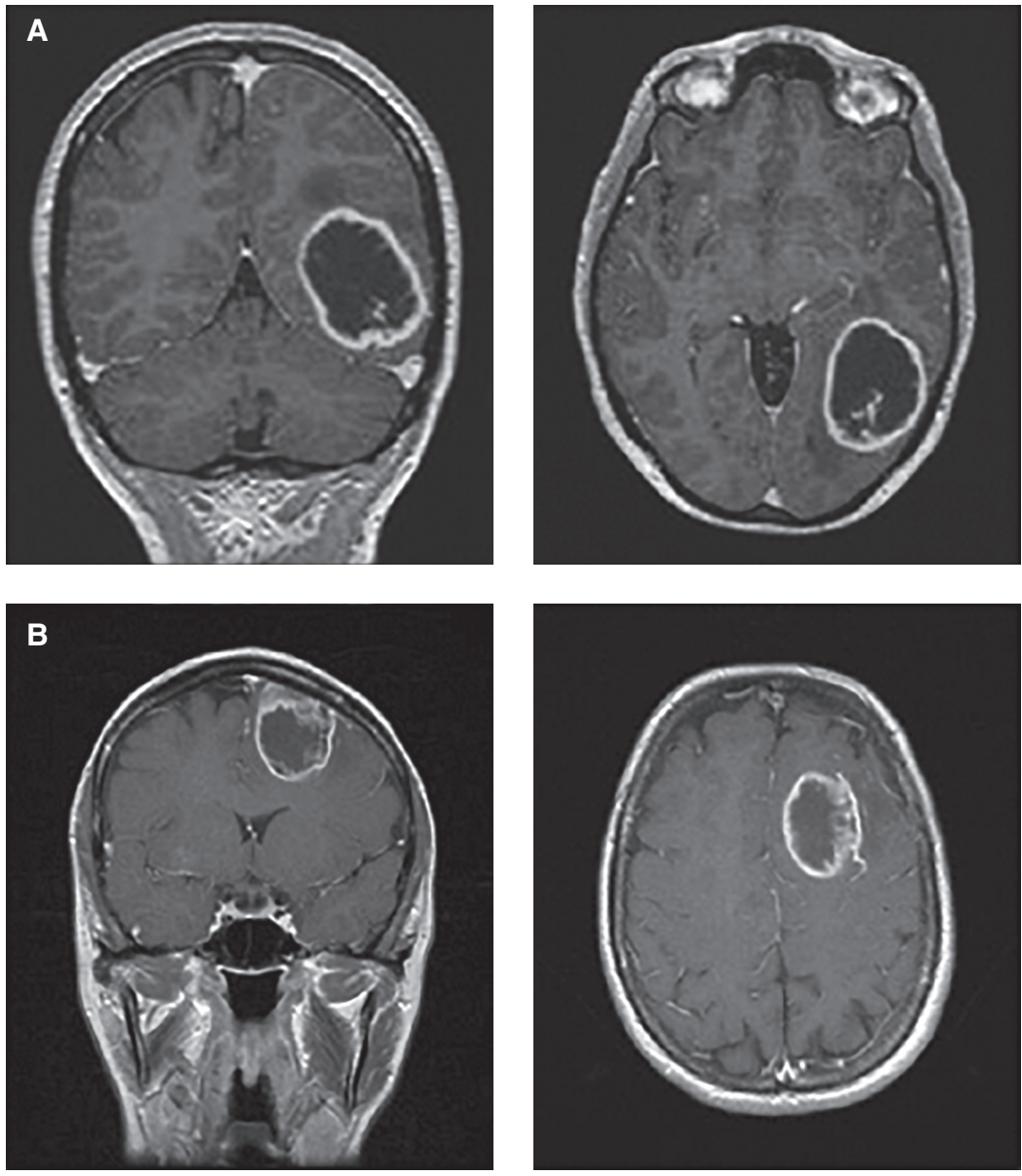

Figure 1. Representative MRI images of the patient's primary and recurrent tumor. T1-weighted MRI images of patient (A) at primary diagnosis (April 2014) (referred to as G89) and (B) at recurrence (September 2016) (referred to as G244).

\section{Molecular Profiling}

$\mathrm{IHC}$ staining of tissues was independently performed by the local pathology laboratory. The proliferation marker Ki67 was relatively high at $50 \%$ for both the primary and recurrent tumors. IHC expression of p53 (>50\%) was also abundant in both tumors. Additionally, IHC staining for PD-1 was performed in-house. Positive PD-1 expression was observed in $<5 \%$ of the whole-cell population. We extracted DNA from fresh frozen tumor tissues and performed pyrosequencing analysis for promoter methylation of the MGMT gene. In both specimens, MGMT promoter methylation was not detected. Both the primary and recurrent tumors were IDH1 wild-type. Lack of mutation in the IDH1 gene was confirmed with both $I H C$ and sequencing. Codeletion of the chromosome arms, $1 p / 19 q$ was absent when confirmed by copy-number (CN) analysis.

WGS was performed with a mean coverage of $>120 \times$ and a tumor purity of $97 \%-100 \%$. Tumor-normal analysis revealed both tumors had high somatic mutation rates at 421 substitutions per megabase (Fig. 2). The primary tumor had 1,336,539 somatic single-nucleotide variants (SNVs) and 168,200 insertion/deletion (indels) mutations (Fig. 2A), whereas the recurrent tumor had 1,336,150 somatic SNVs and 181,756 indels (Fig. 2B). Both tumors had extremely high somatic mutation counts, with 98\% similarity between SNVs and 93\% for 

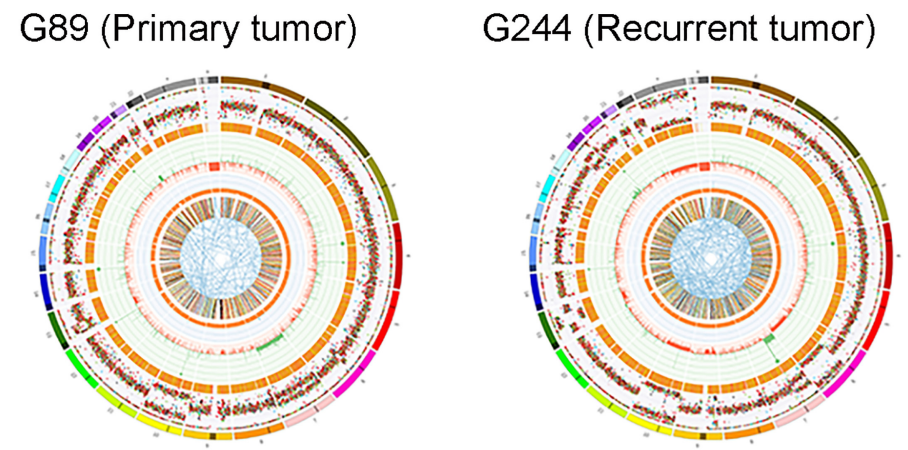

Figure 2. Circos plots and overview of genomic landscape. Circos plots represent chromosomal changes, SNPs, indels, CN changes, minor allele CN changes, and SVs within or between chromosomes of the $(A)$ primary, G89, and (B) recurrent, G244 tumors.

indels, whereas structural variants (SVs) excluding indels shared only $60 \%$ similarity. The primary tumor showed a CN loss on Chromosome 13 and gains in both arms of Chromosome 7, whereas the recurrent tumor had $\mathrm{CN}$ losses on Chromosomes 6, 9, 10, and 13 and $\mathrm{CN}$ gains on Chromosome 19 and only on the p arm of Chromosome 7 (Fig. 2A,B). Of the mutations identified, $4082 \mathrm{SNV}$ s and small indels were found to be potentially damaging in the primary tumor and 4124 in the recurrent. Potentially damaging mutations in this case refer to nonsynonymous, frameshift indels, nonframeshift indels, stop-gain mutations, and stoploss mutations. The mutational landscape of both tumors was determined by identifying the six classes of base pair substitutions, which contained 96 subclassifications based on base pair substitutions (Alexandrov et al. 2013). In both tumor samples, C > T transitions were the most frequently observed, followed by transversions. Mutational signatures observed were signatures 1, 5, and 16 for both tumor samples. SV analysis revealed $60 \%$ of these mutations were identical between the tumors. Bayesian algorithms were used to investigate the clonal architecture of the samples (Miller et al. 2014; Deshwar et al. 2015). Subclonal composition of both tumors was analyzed by examining the variant allele frequencies (VAFs) of somatic $\mathrm{SNV}$ s and $\mathrm{CN}$ variations with clonal mutations defined as being uniformly present in all tumor cells (Fig. 3A,B). Separate analysis of the tumors using PhyloWGS revealed $87.4 \%$ of primary and $80 \%(n=5014)$ of recurrent mutations to be clonal having cancer cell fractions of 1 with 2 and 4 subclonal populations, respectively. When analyzed concurrently, a consensus phylogenetic tree revealed a large clonal cluster (75\% of SNVs) giving rise to three subpopulations. SciClone was also used for two-dimensional clonal visualization in which the clonal cluster is clearly defined surrounded by multiple but less distinct subpopulations (Fig. 3C). The clonal populations maintained mutational signatures of 1, 5, and 16, although the recurrent tumor demonstrated a higher presence of signature 5 with $\mathrm{C}>\mathrm{T}$ transitions and exhibited signature 26, which is associated with defective DNA mismatch repair (MMR) genes. Clonal status of MMR genes was examined in which 11 identical SNVs located in exonic regions or UTRs of MMR genes (MLH1, PMS2, MSH3, MLH3) were found to be clonal in both tumors. Although suspected in certain chromosomes of both tumors, chromothripsis was not detected but more likely caused by areas of homologous repair deficiency.

Cancer driver genes from both the primary and recurrent tumor were identified based on a concatenated reference list of $210 \mathrm{GBM}$ cancer driver and predisposing genes from previous publications (Parsons et al. 2008; Vogelstein et al. 2013). Based on this reference list, two significant nonsynonymous germline mutations of MLH1 (Chromosome 3: 37050343 A/C; rs765014361) and PMS2 (Chromosome 7: 6026775T/C, rs2228006; 6026988 G/A, 
A

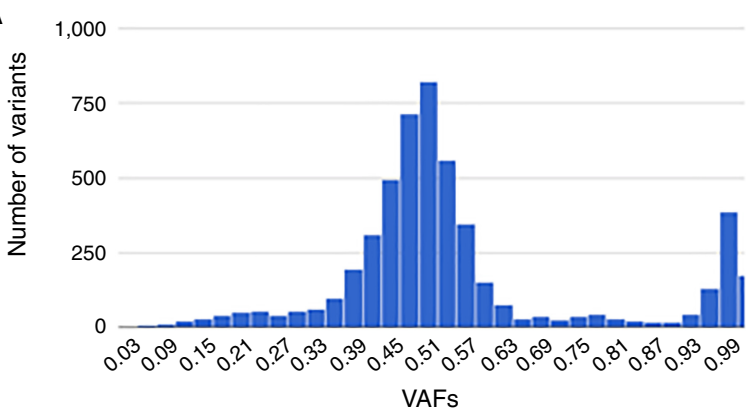

B

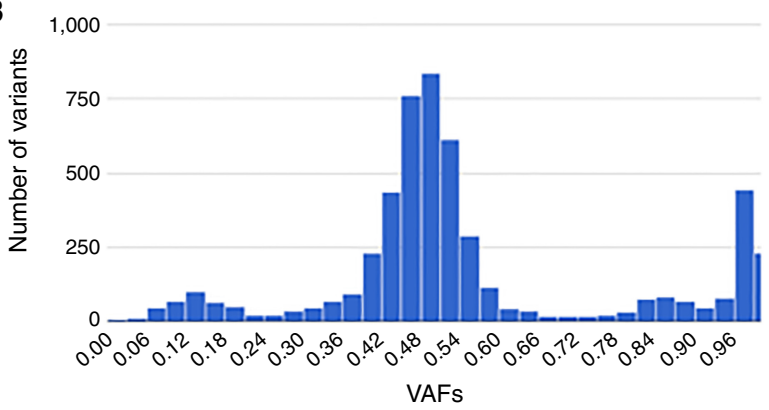

C

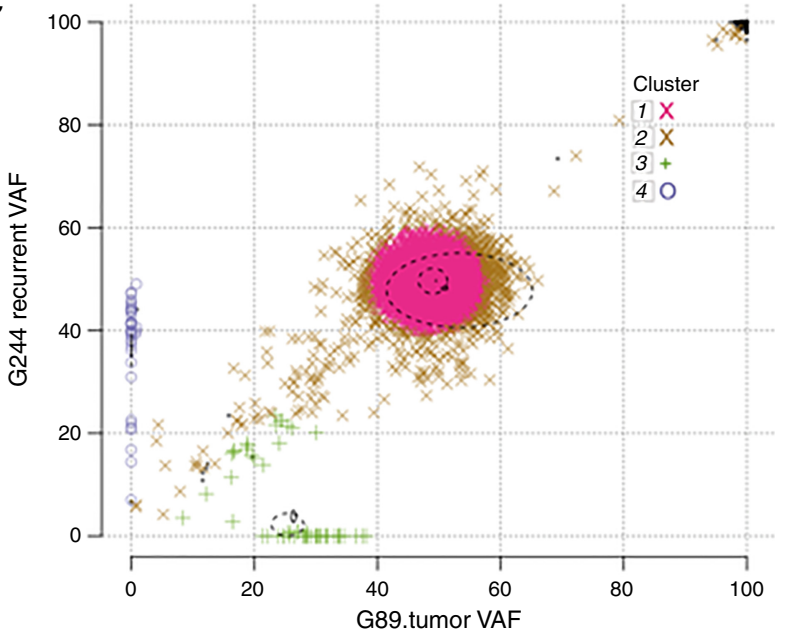

Figure 3. Subclonal analysis. The VAFs are plotted against WGS detected mutations in the (A) primary, G89 and (B) recurrent, G244 tumors. The high-density regions around 50\% VAF indicate expected heterozygous SNVs within the clonal cluster, whereas lower VAFs are indicative of subpopulations. (C) Two-dimensional scatter plot of mutations residing in $\mathrm{CN}$ neutral and loss-of-heterozygosity $(\mathrm{LOH})$ free regions. The $x$-axis represents the VAFs from the primary, G89 tumor and the $y$-axis represents the recurrent, G244 tumor.

rs1805321) were identified, although they remained clinically undetected. MLH1 and PMS2 are MMR genes involved in the repair of insertion/deletion mutations and mis-base incorporation, resulting in a global accumulation of mutations during replication. MLH1 and, to a lesser extent, PMS2 are major drivers of Lynch syndrome, also known as hereditary nonpolyposis colorectal cancer (HNPCC) (Martin-Lopez and Fishel 2013). Lynch syndrome is a type of inherited cancer syndrome associated with a genetic predisposition to various cancer types. For example, lifetime risk for the development of endometrial cancer is $27 \%-71 \%$ (median age at diagnosis: 42-46 yr); gastric cancer 2\%-30\% (median age at diagnosis: 
47-56 yr); cancer of the urinary tract 1\%-28\% (median age at diagnosis: 49-60 yr); and ovarian cancer 3\%-14\% (median age at diagnosis: 40-47 yr). In addition to this, it has been reported that the lifetime risk for the development of brain cancer is $1 \%-4 \%$ (median age at diagnosis: $51 \mathrm{yr}$ ) (Koornstra et al. 2009). GBM is by far the most common variant of brain cancer associated with Lynch syndrome, followed by oligodendrogliomas and ependymomas. In addition, Lynch syndrome-derived brain tumors are not commonly associated with microsatellite instability, a hallmark of many Lynch syndrome cancers (Gylling et al. 2008).

In addition to germline mutations in MLH1 and PMS2, a further 31 somatic cancer driver genes in the primary tumor and 33 cancer driver genes in the recurrent tumor were identified (Supplemental Table 1). Twenty-nine cancer driver genes were shared between the primary and recurrent tumor with the presence of ARIDB1 and HNF1A uniquely observed in the primary tumor and $A B L 1, D S G 4, E G F R$, and RB1 driver mutations uniquely observed in the recurrent tumor. A somatic POLE mutation was observed in both the primary and recurrent tumor and was subsequently confirmed with Sanger sequencing. The POLE mutation observed is an unreported variant causing an E1240K protein change with unknown pathogenicity. Nonsynonymous somatic MMR gene mutations were found in both tumors, MLH1 (I219V), MSH3 (R543S), PMS2 (R563L), MLH3 (P844L), and as germline mutations in saliva, MLH1 (K164N), PMS2 (K435E, P364S) (Table 1).

A network analysis was performed based on the 31 and 33 cancer driver genes observed in the primary and recurrent tumor samples, respectively. Pathway enrichment revealed 106 and 157 interconnected pathways affected in both the primary and recurrent tumor, respectively (Fig. 4A,B). Cluster analysis revealed five major groups of pathways affected in both primary and recurrent tumors. Pathways of interest were selected based on false discovery rates of $<0.05$. Major pathways affected were the PTEN/PI3K/AKT pathway, DNA repair pathway (i.e., Fanconi anemia, MMR, and homologous recombination pathway), the cell cycle pathway, and various signaling pathways (i.e., RTK, GPCR, NOTCH, WNT, hedgehog, MAPK, PTK6, Rho GTPases, EGFR). Of the four unique gene mutations in the recurrent tumor, EGFR appeared predominantly in $23 \%$ of the significantly affected pathways. This finding was also supported by the strong EGFR protein expression positivity in the recurrent tumor.

\begin{tabular}{|c|c|c|c|c|c|c|c|c|}
\hline Gene & Chr & $\begin{array}{l}\text { HGVS DNA } \\
\text { reference }\end{array}$ & $\begin{array}{l}\text { HGVS protein } \\
\text { reference }\end{array}$ & $\begin{array}{l}\text { Variant } \\
\text { type }\end{array}$ & $\begin{array}{c}\text { Predicted effect } \\
\text { (substitution, } \\
\text { deletion, etc.) }\end{array}$ & $\begin{array}{l}\text { dbSNP/ } \\
\text { dbVar ID }\end{array}$ & $\begin{array}{c}\text { Genotype } \\
\text { (heterozygous/ } \\
\text { homozygous) }\end{array}$ & ClinVar ID \\
\hline \multicolumn{9}{|l|}{ Somatic } \\
\hline MLH1 & 3 & c. $655 A>G$ & p.lle219Val & SNV & Nonsynonymous & rs1799977 & Heterozygous & Benign \\
\hline $\mathrm{MSH} 3$ & 5 & c. $1629 \mathrm{G}>\mathrm{C}$ & p.Arg543Ser & SNV & Nonsynonymous & rs780712445 & Heterozygous & Not reported \\
\hline PMS2 & 7 & c. $1688 \mathrm{G}>\mathrm{T}$ & p.Arg563Leu & SNV & Nonsynonymous & rs63750668 & Heterozygous & Likely benign \\
\hline POLE & 12 & c. $3718 \mathrm{G}>\mathrm{A}$ & p.Glu1240Lys & SNV & Nonsynonymous & rs113594027 & Heterozygous & $\begin{array}{l}\text { Uncertain } \\
\text { significance/ } \\
\text { likely benign }\end{array}$ \\
\hline $\mathrm{MLH3}$ & 14 & c. $2531 \mathrm{C}>\mathrm{T}$ & p.Pro844Leu & SNV & Nonsynonymous & rs175080 & Heterozygous & $\begin{array}{l}\text { Benign/likely } \\
\text { benign }\end{array}$ \\
\hline \multicolumn{9}{|c|}{ Germline } \\
\hline MLH1 & 3 & c. $492 \mathrm{~A}>\mathrm{C}$ & p.Lys164Asn & SNV & Nonsynonymous & rs765014361 & Heterozygous & $\begin{array}{l}\text { Uncertain } \\
\text { significance }\end{array}$ \\
\hline PMS2 & 7 & c. $1621 \mathrm{~A}>\mathrm{G}$ & p.Lys541Glu & SNV & Nonsynonymous & rs2228006 & Homozygous & Not reported \\
\hline PMS2 & 7 & c. $1408 C>T$ & p.Pro470Ser & SNV & Nonsynonymous & rs1805321 & Homozygous & Benign \\
\hline
\end{tabular}



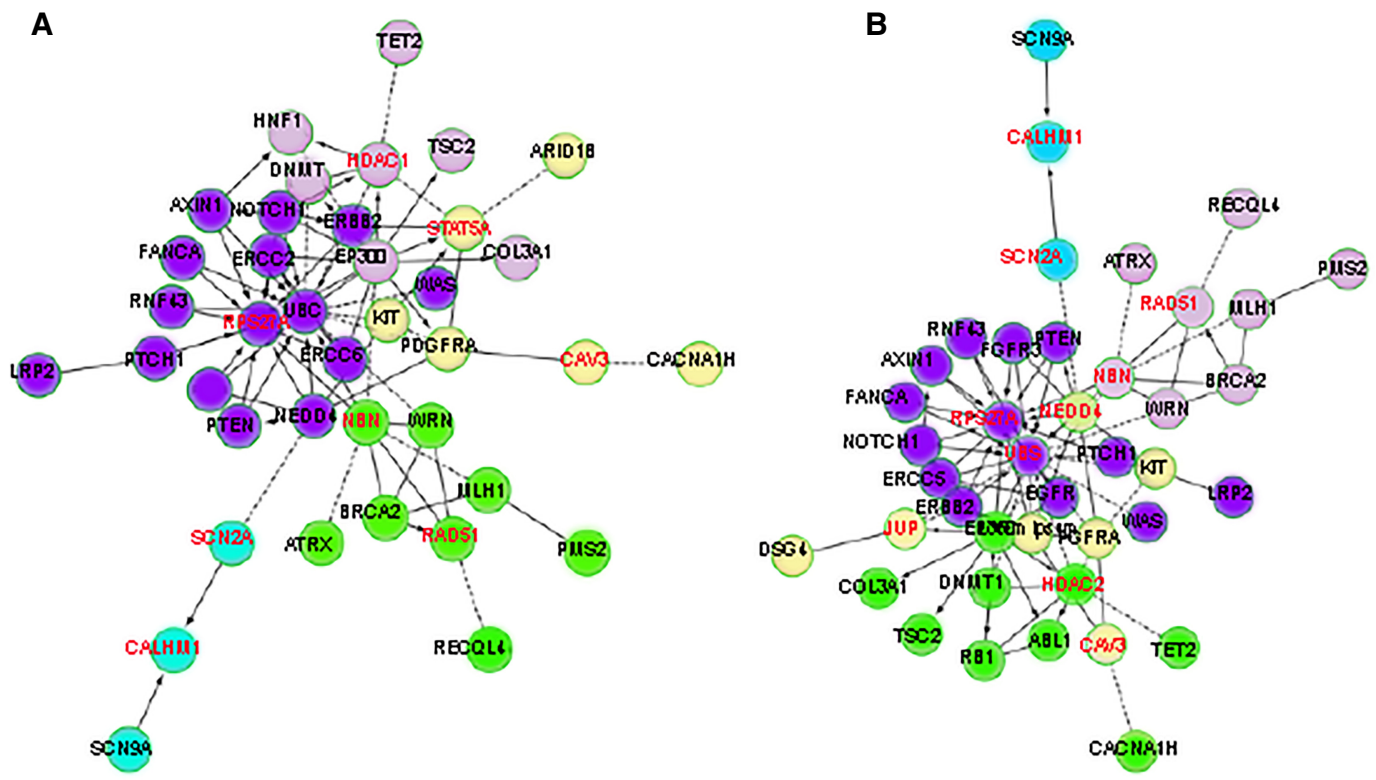

D
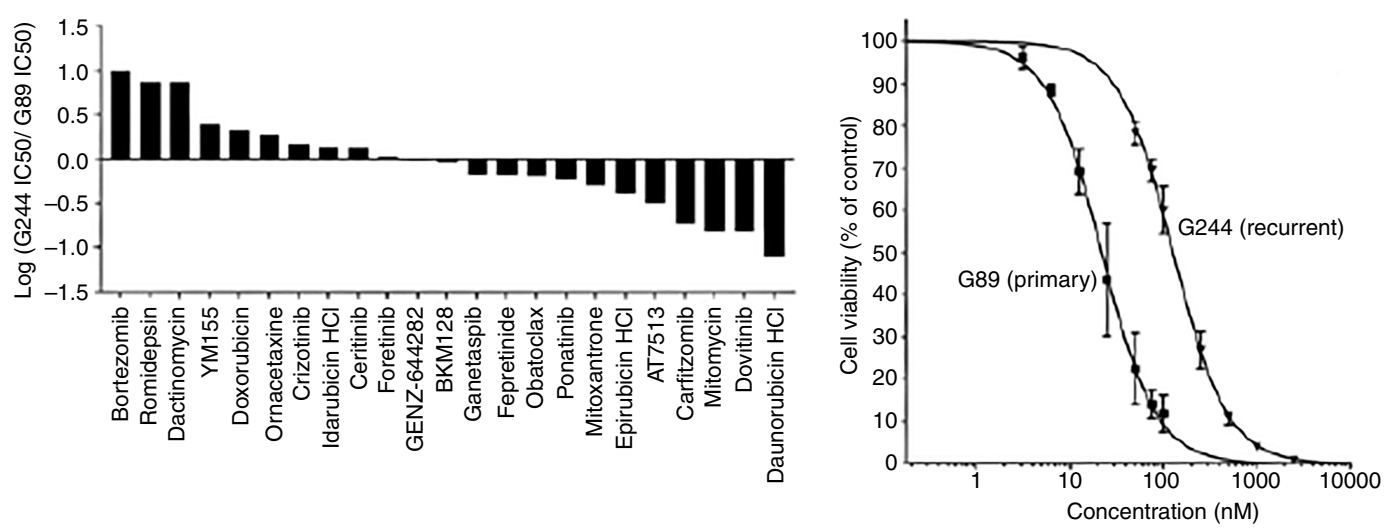

Figure 4. Network analysis and drug screen of patient-derived cells (PDCLs) derived from primary (G89) and recurrent (G244) tumors. Network analysis performed using Reactome FIViz App in cytoscape, which collates information from various pathway databases. The analysis was performed on the 31 and 33 cancer driver genes observed in the (A) primary, G89 and (B) recurrent, G244 tumors. Nodes with black text represent driver genes present in the patient genome. Nodes with red text represent curated linker genes predicted, based on literature, to be connected with actual mutated genes from the patient genome. Black solid lines with arrowheads represent activating or catalyzing interactions between the connected genes. Black broken lines represent predicted functional interactions. A 165-compound screen was performed on PDCLs. The swimmer plot shown in $C$ represents a ratio of the $I C_{50}$ treatment response (Log [recurrent $I C_{50}$ /primary $\left.I C_{50}\right]$ ) between recurrent and primary PDCLs. (D) Dose-response curves for primary (G89) and recurrent (G244) PDCLs treated with increasing doses of the compound, YM155. Recurrent cells (IC50: $21.92 \mathrm{nM}$ ) were more sensitive to YM155 treatment compared to primary cells (IC50: $127.30 \mathrm{nM}$ ).

\section{Drug Screening Panel}

A drug screening assay comprising a panel of 165 compounds (Supplemental Table 2) were tested on both the primary and recurrent PDCLs. Differential sensitivity to multiple drugs were observed between the primary and recurrent PDCL (Fig. 4C). Of the 165 compounds 
we found 28 compounds with an $\mathrm{IC}_{50}$ of $<5 \mu \mathrm{M}$ and six compounds that had an $\mathrm{IC}_{50}$ of $<0.05$ $\mu \mathrm{M}$ in the primary PDCL. In contrast, the recurrent PDCL responded to 35 compounds with an $\mathrm{IC}_{50}$ of $<5 \mu \mathrm{M}$, and 10 compounds with an $\mathrm{IC}_{50}$ of $<0.05 \mu \mathrm{M}$. Unfortunately, upon further scrutiny of the compounds, only one of the candidate treatments crossed the blood-brain barrier. YM155, a survivin inhibitor, is an investigational compound that has been previously tested in clinical trials of other cancer types. YM155 has shown efficacy in GBM preclinical models (Lai et al. 2012; Householder et al. 2015). We tested this compound on both the primary and recurrent tumor specimens and observed favorable response (Fig. 4D).

\section{DISCUSSION}

WGS of patient G89 and her recurrent tumor G244 revealed a striking mutation pattern at a rate of 421 substitutions per megabase. Melanoma, lung, stomach, colorectal, endometrial, and cervical cancers display high mutation loads consistent with hypermutation, which may generate drivers of malignancy. In contrast, hypermutation is a rare observation in patients diagnosed with GBM, occurring in $~ 1 \%$ of patients diagnosed with the disease (ErsonOmay et al. 2015; Hodges et al. 2017). What was striking about this patient case was that a de novo hypermutation phenotype was observed in the TMZ-naive tumor. TMZ-naive hypermutated tumors were marked by absence of IDH1 somatic mutation and MGMT gene promoter methylation, two genomic traits that were significantly associated with the TMZ-induced hypermutagenic event in GBM and harbored inherited alterations in the MMR machinery (Sa et al. 2018). MLH1 and PMS2 are both members of the MMR system, and their disruption contributes to the hypermutated genotype associated with the onset of Lynch syndrome.

The saliva DNA of the patient was shown to have a germline heterozygotic mutation in the gene MLH1. The deleterious effects of this particular mutation were considered of unknown significance, as assessed with ClinVar, and are undocumented in the literature (Landrum et al. 2018). Given that this mutation is nonsynonymous, occurring in the coding region and changing the amino acid sequence $(\mathrm{K} 146 \mathrm{~N})$, this mutation is likely pathogenic. Although this mutation is heterozygotic and tolerable, a "second hit" of the adjacent allele usually follows, often via MLH1 promoter methylation, which leads to the onset of Lynch syndrome. Xia and colleagues found the prevalence of MLH1 promoter methylation in Lynch syndrome-associated colorectal cancer at $>16 \%$, suggesting that this could be the "second hit," given that the mutation remained heterozygous (Li et al. 2013). Loss of the mutant allele in both tumors could be further explained by loss of heterozygosity (LOH). Ollikainen and colleagues, reported that in a cohort of 57 patients with Lynch syndrome, 31 (54.4\%), displayed LOH of one allele (Ollikainen et al. 2007). The prevalence of LOH of MLH1 in Lynch syndrome suggests that the methylated MLH1 allele substituted the mutated allele (rs765014361 A/C) while still leaving the methylated MLH1 alleles nonfunctional. Given the saliva DNA of the patient displayed a hypermutation profile, it is likely that this mutation played a contributing factor. The patient in this study also displayed two nonsynonymous germline MMR mutations in the gene PMS2 (K435E, P364S), which is also associated with Lynch syndrome. However, these mutations were considered benign by ClinVar, although their actual effects cannot be discounted. As such, the patient put forward in this study most likely had Lynch syndrome, which may have contributed to her hypermutation and development of brain cancer.

Somatic mutations in the POLE gene, found in both the primary and recurrent tumors of the patient, may also contribute to the high mutational burden and prolonged survival time. The POLE gene plays an important role in maintaining the organization of chromosomal DNA replication (Guerra et al. 2017). Somatic mutations in the POLE gene have been 
attributed to longer survival in colorectal and endometrial cancers (Church et al. 2013; Ahn et al. 2016). POLE mutations were associated with a hypermutated somatic genotype and extended progression-free survival in giant cell GBM (Erson-Omay et al. 2015; Johanns et al. 2016). Mutations in POLE have been demonstrated to interfere with the proofreading capabilities of DNA polymerase. It is speculated that the burden induced by the mutation of POLE, combined with disruption of the MMR system, may prevent purposeful and optimal tumor evolution and may promote survival in such instances (Kim et al. 2013; Meng et al. 2014). We have presented a schematic overview in Figure 5.

Tumor hypermutation has been proposed as a potential biomarker for immune therapies (Goodman et al. 2017). Hypermutated tumors often have higher levels of neoantigens that can be recognized by the immune system (Maleki Vareki 2018). In a large cohort study of GBM, hypermutated tumors were associated with a higher influx of CD8 ${ }^{+}$cells (Wang et al. 2017). However, emerging data continues to challenge the association between mutational load and antitumor response to immune checkpoint blockade (Jia et al. 2018). For example, in the CheckMate 026 NSCLC trial, mutational load was not correlated with PD-L1 expression (Carbone et al. 2017). Furthermore, only 62\% of patients with dMMR status experienced a clinical benefit to immune checkpoint blockade (Le et al. 2015). The patient at the center of this current case was treated with pembrolizumab, a PD-1 checkpoint inhibitor. The patient only received four cycles because of progression and reoperation. However, given that only $5 \%$ of tumor cells demonstrated PD-1 expression, it was unlikely that the PD-1 inhibitor, pembrolizumab, would display any efficacy.

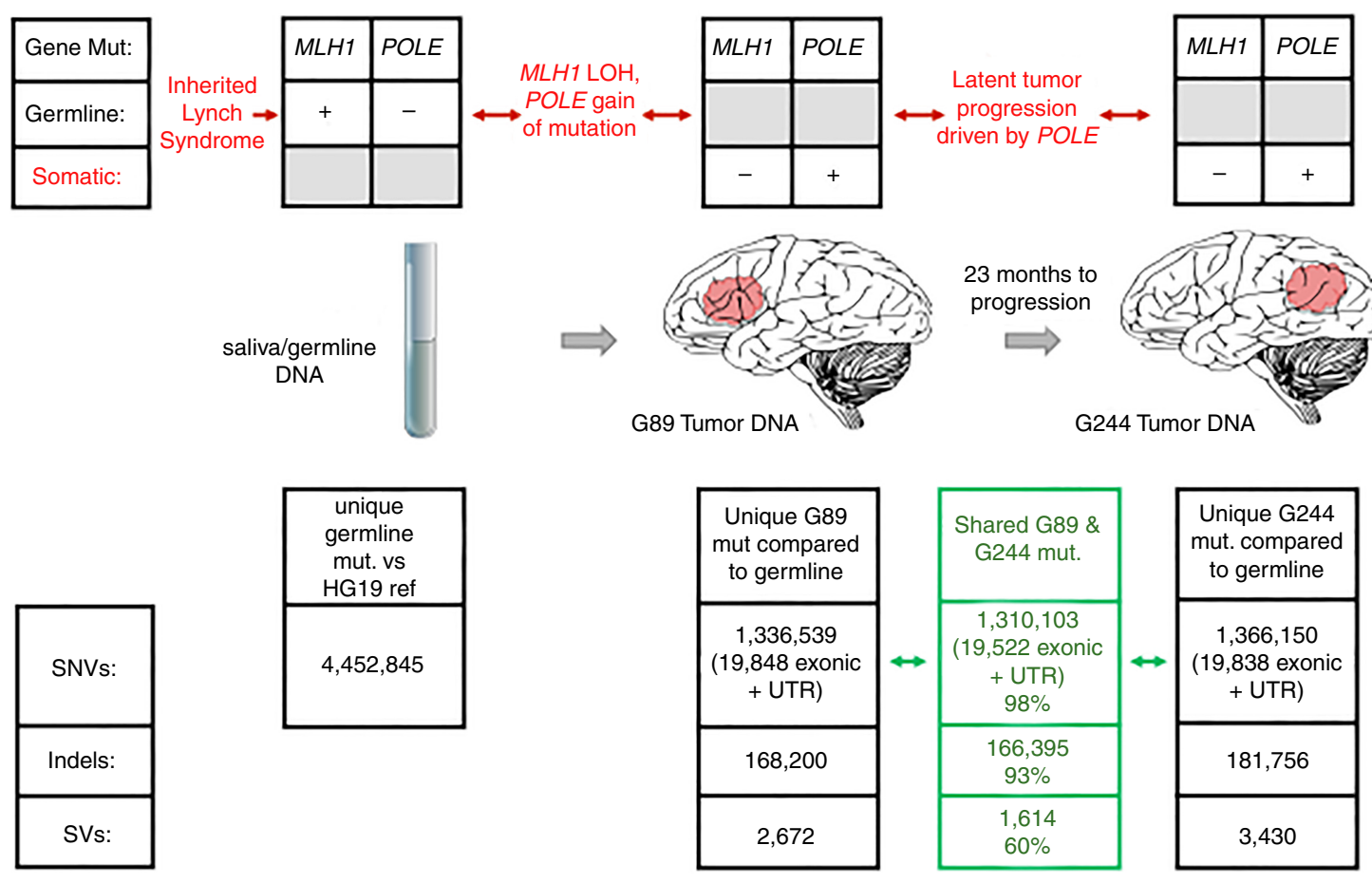

Figure 5. A schematic representation of tumor development and evolution. A germline mutation of MLH1 followed by LOH is presumed to give rise to a hypermutated genotype and onset of Lynch syndrome. A further somatic mutation of POLE in both primary and recurrent tumor may have sufficiently disrupted DNA repair to limit positive tumor evolution and prolong survival. Total combined single-nucleotide variant (SNV), insertion/ deletion (indel), and structural variant (SV) mutations are displayed for the germline saliva DNA and primary and recurrent tumors, with conservation of mutations between tumors displayed. (UTR) Untranslated region. 
A distinct advantage of this case study was the availability of fresh tumor from the primary (treatment naive) resection and the recurrent (treated with RT/TMZ) resection. The recurrent lesion was distally located. Surprisingly, a high similarity (98\%) was observed in the small variant somatic genomic landscape of both the primary and recurrent tumor. This is in contrast to previous studies in which it was observed that distantly recurring tumor growth showed a considerable change to its genetic makeup in comparison to the primary tumor (Kim et al. 2015b). Although we are uncertain as to why smaller variants were highly conserved, appreciable differences were observed when considering the larger genomic rearrangements such as inversions, translocations, and insertions. The recurrent tumor harbored 760 more $\mathrm{SVs}$ and consequentially experienced greater $\mathrm{CN}$ loss throughout its genome. Because of the intratumoral heterogeneic nature of GBM, a high mutational overlap between primary and recurrent counterparts is not indicative of identical clonal architecture. However, in this particular case the overlapping mutational landscape shared the same subclonal lineage as indicated by the consensus phylogenetic tree and two-dimensional analysis of the tumors (Fig. 3). Further investigation into MMR mutations residing in exonic regions and UTRs found all to be clonal, a result differing from the findings of Kim and colleagues who found MMR mutations of recurrent GBM tumors to be subclonal (Kim et al. 2015a).

Network analysis revealed more than 100 interconnected pathways affected by mutations from identified driver genes from the patient genome. We identified mTOR and PARP as possible targets of interest; however, in vitro experimentations did not result in a significant positive outcome (data not shown). EGFR was observed to be predominant in $23 \%$ of the affected pathways and was correlated to a high positive staining for EGFR on IHC; hence, an EGFR inhibitor (ABT-414) was recommended and commenced. However, there was no evidence of clinical benefit. A drug screen performed on a panel of $165 \mathrm{com}-$ pounds found just one drug agent that elicited high sensitivity to the PDCLs, which crossed the blood-brain barrier; however, the drug was experimental and was not readily available to the patient.

In summary, we have presented a complex case of a hypermutated GBM (both treated and treatment-naive) that demonstrated long-term progression-free and overall survival. The hypermutated phenotype was most likely a result of the patients' undiagnosed Lynch syndrome; however, the presence of mutations in POLE may also have contributed while simultaneously conferring a survival benefit. Despite the spatially distal location of the recurrent tumor, typical clonal evolution was not observed. This case report demonstrates the utility of sequencing at the time of diagnosis and subsequent recurrence.

\section{METHODS}

\section{Patient Tissue Processing}

Fresh tumor tissue from the two surgeries-primary and recurrent-was collected by the laboratory within $15 \mathrm{~min}$ of the excision. The tissues were dissected and (1) flash frozen in liquid nitrogen for molecular analysis, (2) put into PAX gene containers as a source for paraffinembedded tissue, and (3) dissociated into single cells to establish patient-derived preclinical models.

\section{Patient-Derived Preclinical Models}

Patient-derived cell lines (PDCLs) from the primary and recurrent tumor were grown in RHB-A medium (Clontech Laboratories, Inc.) supplemented with human Epidermal Growth Factor $(20 \mathrm{ng} / \mathrm{mL}$; Sigma-Aldrich) and human Fibroblast Growth Factor-Basic $(20 \mathrm{ng} / \mathrm{mL}$; SigmaAldrich) in tissue culture flasks coated with a layer of BD Matrigel Basement Membrane 
COLD SPRING HARBOR Molecular Case Studies
Using genomic data to personalize treatment
Matrix (1:100 in PBS; BD Biosciences). Cells were maintained in a $37^{\circ} \mathrm{C}, 5 \% \mathrm{CO}_{2}$ incubator (Thermo Fisher Scientific).

\section{MGMT Promoter Methylation}

The percentage level of MGMT promoter methylation was assessed by CpG pyrosequencing after a bisulfite modification step as previously described (McDonald et al. 2013). Briefly, $500 \mathrm{ng}$ of tumor DNA was bisulfite modified using the EZ DNA methylation kit (Zymo Research) according to the manufacturer's recommendations. The pyrosequencing assay was performed using the PyroMark MGMT kit (QIAGEN) on a PSO 24 MA system (QIAGEN) according to the manufacturer's protocol. Pyromark CpG software (QIAGEN) was used to quantify the methylation in the samples. A cutoff of $9 \%$ was used to determine the methylation status of the samples.

\section{Whole-Genome Sequencing}

DNA was extracted from fresh frozen tumor tissue and saliva (germline DNA) using QIAmp DNA Mini Kit (QIAGEN) following the manufacturer's instructions. For whole tumor tissue, the tissue was homogenized using a 23G syringe needle while suspended in the lysis buffer provided in the kit. DNA concentration was quantified using the NanoDrop ND-1000 spectrophotometer (Thermo Fisher Scientific). DNA specimens were sent to the Kinghorn Centre for Clinical Genomics (KCCG) and paired-end (2×150 bp) WGS was performed on the Illumina HiSeq X Ten Sequencing platform using TruSeq DNA Nano v2.5.

The sequencing reads were aligned to the reference human genome (hg19) using the Burrows-Wheeler Aligner v0.7.17 (BWA mem) (Li and Durbin 2010). Once aligned, PCR duplicates were marked and base quality recalibration conducted according to Genome Analysis Toolkit's Best Practices v4.0 (McKenna et al. 2010; Van der Auwera et al. 2013). Sequencing coverage for each sample is shown in Supplemental Table 3.

Sequence alignment files (BAM files) underwent variant analysis following Hartwig Medical Foundation's hmftools (Priestley et al. 2018). Utilities of hmftools included the use of Strelka v2.9.7 for single-nucleotide variant and small indel variant discovery using Strelka's filtering parameters to extract high-confidence variant calls (Saunders et al. 2012). Variants that satisfied filters underwent functional annotation using ANNOVAR v2018-0416 and Variant Effector Predictor v95.0 (Wang et al. 2010; McLaren et al. 2016). The ClinVar, SIFT, and PolyPhen algorithms were used to predict the functional impact of an SNV (Kumar et al. 2009; Adzhubei et al. 2010; Landrum et al. 2018). Manta v1.4.0 was used for SV discovery followed by hmftools break-point-inspector (BPI) (Chen et al. 2016). BPI reanalyzed Manta calls, thereby removing false positives through multiple filters and extracting high confidence SV calls.

\section{Tumor Purity Estimation}

Hmftools' purity-ploidy-estimator v2.16 was used to calculate the purity and CN profile of each sample.

\section{Data Visualization}

To visualize the WGS analysis data, Circos plots were generated using Circos v0.69-6 (Krzywinski et al. 2009). Clonal status was analyzed using PhyloWGS v3.0 and SciClone v1.1.0 (Miller et al. 2014; Deshwar et al. 2015), mutational signatures using deconstructSigs v1.8.0 (Rosenthal et al. 2016), and chromothripsis using Shatterseek v0.4 (Cortés-Ciriano et al. 2018). 
COLD SPRING HARBOR Molecular Case Studies
Using genomic data to personalize treatment

\section{Network Analysis}

Network analysis was performed to determine the molecular network affected by specific gene mutations observed in the patient's genome. The analysis was performed by initially screening for cancer driver genes against a reference list concatenated from previous publications (Parsons et al. 2008; Vogelstein et al. 2013). Mutated cancer driver genes were analyzed using the pathway enrichment and cluster analysis function in the Reactome FIViz app version 2016 in Cytoscape version 3.5.1.

\section{Drug Screening Panel}

For the drug screening panel, PDCLs were seeded at 2000 cells per well in a 384-well plate coated with $0.1 \%$ gelatin. Cells were allowed to attach for $24 \mathrm{~h}$ prior to a 72 -h drug exposure. Cells were treated with five different concentrations $(0.5 \mathrm{nM}-5 \mu \mathrm{M})$ in 1:10 fold dilutions for each compound. Treated cells were incubated at $37^{\circ} \mathrm{C}$ in a $5 \% \mathrm{CO}_{2}$ humidified incubator. Cell viability was analyzed using Cell-Titer Glo (Promega), a luciferase-based cell viability assay. The drug screening panel included 165 compounds. Difference in response was assessed by calculating for the log of the ratio between the $\mathrm{IC}_{50}$ values of the recurrent over the primary PDCLs.

For validation of the selected drug, cells were seeded in 96-well plates and treated with seven different concentrations. Plated cells were then incubated for $72 \mathrm{~h}$ at $37^{\circ} \mathrm{C}$ in a $5 \% \mathrm{CO}_{2}$ humidified incubator. Cell viability was measured using the MTS assay.

\section{ADDITIONAL INFORMATION}

\section{Data Deposition and Access}

The MLH1 variant interpreted in this study was submitted to Clinvar (https://www.ncbi.nlm .nih.gov/clinvar/) and can be found under accession number SCV000902257. Raw sequencing data have been submitted to NCBI SRA (https://www.ncbi.nlm.nih.gov/sra) under submission number SUB5583293.

\section{Ethics Statement}

The patient was enrolled in a Human Research Ethics Committee (HREC)-approved human study at the University of New South Wales (Sydney, Australia; HREC 10/121) and consented to publication of photos and their clinical data.

\section{Author Contributions}

T.R.J. and L.R.O. equally contributed to the drafting and revision of the manuscript. T.R.J. performed in vitro and in vivo experiments, data analysis, and manuscript drafting and revision. L.R.O. performed the majority of the data analysis (bioinformatics) and manuscript revision. A.H.S. and M.D. performed in vitro experiments. R.W.R., V.M.L., S.A.C., and S.P.W. provided intellectual input in the data analysis and in the drafting of the manuscript. B.N. and J.X.M.Y. performed data analysis for the bioinformatic component of the manuscript. E.J.H. (oncologist) and J.M.F. (surgeon) were the clinicians caring for the patient discussed in this study. K.L.M. provided overall guidance to the success of the study as well as the drafting of the manuscript.

This study has been supported by the Cure Brain Cancer Foundation.

\section{Funding}

Received August 26, 2018; accepted in revised form March 25, 2019
Competing Interest Statement

The authors have declared no competing interest.

Jue et al. 2019 Cold Spring Harb Mol Case Stud 5: a003251 


\section{REFERENCES}

Adzhubei IA, Schmidt S, Peshkin L, Ramensky VE, Gerasimova A, Bork P, Kondrashov AS, Sunyaev SR. 2010. A method and server for predicting damaging missense mutations. Nat Methods 7: 248-249. doi:10.1038/ nmeth0410-248

Ahn SM, Ansari AA, Kim J, Kim D, Chun SM, Kim J, Kim TW, Park I, Yu CS, Jang SJ. 2016. The somatic POLE P286R mutation defines a unique subclass of colorectal cancer featuring hypermutation, representing a potential genomic biomarker for immunotherapy. Oncotarget 7: 68638-68649. doi:10.18632/oncotarget .11862

Alexandrov LB, Nik-Zainal S, Wedge DC, Aparicio SAJR, Behjati S, Biankin AV, Bignell GR, Bolli N, Borg A Børresen-Dale AL, et al. 2013. Signatures of mutational processes in human cancer. Nature 500: 415421. doi:10.1038/nature12477

Carbone DP, Reck M, Paz-Ares L, Creelan B, Horn L, Steins M, Felip E, van den Heuvel MM, Ciuleanu TE, Badin $F$, et al. 2017. First-line nivolumab in stage IV or recurrent non-small-cell lung cancer. N Engl J Med 376: 2415-2426. doi:10.1056/NEJMoa1613493

Chen X, Schulz-Trieglaff O, Shaw R, Barnes B, Schlesinger F, Källberg M, Cox AJ, Kruglyak S, Saunders CT. 2016. Manta: rapid detection of structural variants and indels for germline and cancer sequencing applications. Bioinformatics 32: 1220-1222. doi:10.1093/bioinformatics/btv710

Church DN, Briggs SE, Palles C, Domingo E, Kearsey SJ, Grimes JM, Gorman M, Martin L, Howarth KM, Hodgson SV, et al. 2013. DNA polymerase $\varepsilon$ and $\delta$ exonuclease domain mutations in endometrial cancer. Hum Mol Genet 22: 2820-2828. doi:10.1093/hmg/ddt131

Cortés-Ciriano I, Lee J-K, Xi R, Jain D, Jung YL, Yang L, Gordenin D, Klimczak LJ, Zhang CZ, Pellman DS, et al. 2018. Comprehensive analysis of chromothripsis in 2,658 human cancers using whole-genome sequencing. bioRxiv doi:10.1101/333617

Deshwar AG, Vembu S, Yung CK, Jang GH, Stein L, Morris Q. 2015. PhyloWGS: reconstructing subclonal composition and evolution from whole-genome sequencing of tumors. Genome Biol 16: 35. doi:10.1186/ s13059-015-0602-8

Erson-Omay EZ, Çağlayan AO, Schultz N, Weinhold N, Omay SB, Özduman K, Köksal Y, Li J, Serin Harmancı A, Clark V, et al. 2015. Somatic POLE mutations cause an ultramutated giant cell high-grade glioma subtype with better prognosis. Neuro Oncol 17: 1356-1364. doi:10.1093/neuonc/nov027

Goodman AM, Kato S, Bazhenova L, Patel SP, Frampton GM, Miller V, Stephens PJ, Daniels GA, Kurzrock R. 2017. Tumor mutational burden as an independent predictor of response to immunotherapy in diverse cancers. Mol Cancer Ther 16: 2598-2608. doi:10.1158/1535-7163.Mct-17-0386

Guerra J, Pinto C, Pinto D, Pinheiro M, Silva R, Peixoto A, Rocha P, Veiga I, Santos C, Santos R, et al. 2017. POLE somatic mutations in advanced colorectal cancer. Cancer Med 6: 2966-2971. doi:10.1002/cam4 .1245

Gylling AH, Nieminen TT, Abdel-Rahman WM, Nuorva K, Juhola M, Joensuu El, Järvinen HJ, Mecklin JP, Aarnio M, Peltomäki PT. 2008. Differential cancer predisposition in Lynch syndrome: insights from molecular analysis of brain and urinary tract tumors. Carcinogenesis 29: 1351-1359. doi:10.1093/carcin/bgn133

Hodges TR, Ott M, Xiu J, Gatalica Z, Swensen J, Zhou S, Huse JT, de Groot J, Li S, Overwijk WW, et al. 2017. Mutational burden, immune checkpoint expression, and mismatch repair in glioma: implications for immune checkpoint immunotherapy. Neuro Oncol 19: 1047-1057. doi:10.1093/neuonc/nox026

Householder KT, DiPerna DM, Yamaguchi JT, Sanai N, Mehta S, Sirianni RW. 2015. Abstract B21: use of polymeric nanoparticles for the delivery of YM155 to glioma cells in vitro and in vivo. Cancer Res 75: B21. doi:10 .1158/1538-7445.brain15-b21

Jia Q, Wu W, Wang Y, Alexander PB, Sun C, Gong Z, Cheng JN, Sun H, Guan Y, Xia X, et al. 2018. Local mutational diversity drives intratumoral immune heterogeneity in non-small cell lung cancer. Nat Commun 9: 5361. doi:10.1038/s41467-018-07767-w

Johanns TM, Miller CA, Dorward IG, Tsien C, Chang E, Perry A, Uppaluri R, Ferguson C, Schmidt RE, Dahiya S, et al. 2016. Immunogenomics of hypermutated glioblastoma: a patient with germline POLE deficiency treated with checkpoint blockade immunotherapy. Cancer Discov 6: 1230-1236. doi:10.1158/21598290.cd-16-0575

Jue TR, McDonald KL. 2016. The challenges associated with molecular targeted therapies for glioblastoma. J Neuro Oncol 127: 427-434. doi:10.1007/s11060-016-2080-6

Kim HR, Kim HC, Yun HR, Kim SH, Park CK, Cho YB, Yun SH, Lee WY, Chun HK. 2013. An alternative pathway in colorectal carcinogenesis based on the mismatch repair system and p53 expression in Korean patients with sporadic colorectal cancer. Ann Surg Oncol 20: 4031-4040.

Kim H, Zheng S, Amini SS, Virk SM, Mikkelsen T, Brat DJ, Grimsby J, Sougnez C, Muller F, Hu J, et al. 2015a. Whole-genome and multisector exome sequencing of primary and post-treatment glioblastoma reveals patterns of tumor evolution. Genome Res 25: 316-327. doi:10.1101/gr.180612.114 
Kim J, Lee IH, Cho HJ, Park CK, Jung YS, Kim Y, Nam SH, Kim BS, Johnson MD, Kong DS, et al. 2015b. Spatiotemporal evolution of the primary glioblastoma genome. Cancer Cell 28: 318-328. doi:10.1016/j .ccell.2015.07.013

Koornstra JJ, Mourits MJ, Sijmons RH, Leliveld AM, Hollema H, Kleibeuker JH. 2009. Management of extracolonic tumours in patients with Lynch syndrome. Lancet Oncol 10: 400-408. doi:10.1016/S1470-2045(09) 70041-5

Krzywinski M, Schein J, Birol I, Connors J, Gascoyne R, Horsman D, Jones SJ, Marra MA. 2009. Circos: an information aesthetic for comparative genomics. Genome Res 19: 1639-1645. doi:10.1101/gr.092759.109

Kumar P, Henikoff S, Ng PC. 2009. Predicting the effects of coding non-synonymous variants on protein function using the SIFT algorithm. Nat Protoc 4: 1073-1081. doi:10.1038/nprot.2009.86

Lai PC, Chen SH, Yang SH, Cheng CC, Chiu TH, Huang YT. 2012. Novel survivin inhibitor YM155 elicits cytotoxicity in glioblastoma cell lines with normal or deficiency DNA-dependent protein kinase activity. Pediatr Neonatol 53: 199-204. doi:10.1016/j.pedneo.2012.04.008

Landrum MJ, Lee JM, Benson M, Brown GR, Chao C, Chitipiralla S, Gu B, Hart J, Hoffman D, Jang W, et al. 2018. ClinVar: improving access to variant interpretations and supporting evidence. Nucleic Acids Res 46: D1062-D1067. doi:10.1093/nar/gkx1153

Le DT, Uram JN, Wang H, Bartlett BR, Kemberling H, Eyring AD, Skora AD, Luber BS, Azad NS, Laheru D, et al. 2015. PD-1 blockade in tumors with mismatch-repair deficiency. N Engl J Med 372: 2509-2520. doi:10 .1056/NEJMoa1500596

Li H, Durbin R. 2010. Fast and accurate long-read alignment with Burrows-Wheeler transform. Bioinformatics 26: 589-595. doi:10.1093/bioinformatics/btp698

Li X, Yao X, Wang Y, Hu F, Wang F, Jiang L, Liu Y, Wang D, Sun G, Zhao Y. 2013. MLH1 promoter methylation frequency in colorectal cancer patients and related clinicopathological and molecular features. PLoS One 8: e59064. doi:10.1371/journal.pone.0059064

Maleki Vareki S. 2018. High and low mutational burden tumors versus immunologically hot and cold tumors and response to immune checkpoint inhibitors. J Immunother Cancer 6: 157. doi:10.1186/s40425-0180479-7

Martin-Lopez JV, Fishel R. 2013. The mechanism of mismatch repair and the functional analysis of mismatch repair defects in Lynch syndrome. Fam Cancer 12: 159-168. doi:10.1007/s10689-013-9635-x

McDonald KL, Rapkins RW, Olivier J, Zhao L, Nozue K, Lu D, Tiwari S, Kuroiwa-Trzmielina J, Brewer J, Wheeler $H R$, et al. 2013. The T genotype of the MGMT C>T (rs16906252) enhancer single-nucleotide polymorphism (SNP) is associated with promoter methylation and longer survival in glioblastoma patients. Eur $J$ Cancer 49: 360-368. doi:10.1016/j.ejca.2012.08.012

McKenna A, Hanna M, Banks E, Sivachenko A, Cibulskis K, Kernytsky A, Garimella K, Altshuler D, Gabriel S, Daly M, et al. 2010. The Genome Analysis Toolkit: a MapReduce framework for analyzing next-generation DNA sequencing data. Genome Res 20: 1297-1303. doi:10.1101/gr.107524.110

McLaren W, Gil L, Hunt SE, Riat HS, Ritchie GR, Thormann A, Flicek P, Cunningham F. 2016. The Ensembl Variant Effect Predictor. Genome Biol 17: 122. doi:10.1186/s13059-016-0974-4

Meng B, Hoang LN, McIntyre JB, Duggan MA, Nelson GS, Lee CH, Köbel M. 2014. POLE exonuclease domain mutation predicts long progression-free survival in grade 3 endometrioid carcinoma of the endometrium. Gynecol Oncol 134: 15-19. doi:10.1371/journal.pcbi.1003665

Miller CA, White BS, Dees ND, Griffith M, Welch JS, Griffith OL, Vij R, Tomasson MH, Graubert TA, Walter MJ, et al. 2014. SciClone: inferring clonal architecture and tracking the spatial and temporal patterns of tumor evolution. PLoS Comput Biol 10: e1003665. doi:10.1371/journal.pcbi.1003665

Minniti G, Muni R, Lanzetta G, Marchetti P, Enrici RM. 2009. Chemotherapy for glioblastoma: current treatment and future perspectives for cytotoxic and targeted agents. Anticancer Res 29: 5171-5184.

Ollikainen M, Hannelius U, Lindgren CM, Abdel-Rahman WM, Kere J, Peltomaki P. 2007. Mechanisms of inactivation of MLH1 in hereditary nonpolyposis colorectal carcinoma: a novel approach. Oncogene 26: 4541-4549. doi:10.1038/sj.onc.1210236

Parsons DW, Jones S, Zhang X, Lin JC, Leary RJ, Angenendt P, Mankoo P, Carter H, Siu IM, Gallia GL, et al. 2008. An integrated genomic analysis of human glioblastoma multiforme. Science 321: 1807. doi:10 $.1126 /$ science. 1164382

Priestley P, Baber J, Lolkema M, Steeghs N, de Bruijn E, Duyvesteyn K, Haidari S, van Hoeck A, Onstenk W, Roepman P, et al. 2018. Pan-cancer whole genome analyses of metastatic solid tumors. bioRxiv doi:10 $.1101 / 415133$

Rosenthal R, McGranahan N, Herrero J, Taylor BS, Swanton C. 2016. DeconstructSigs: delineating mutational processes in single tumors distinguishes DNA repair deficiencies and patterns of carcinoma evolution. Genome Biol 17: 31. doi:10.1186/s13059-016-0893-4

Sa JK, Choi SW, Zhao J, Lee Y, Zhang J, Kong DS, Choi JW, Seol HJ, Lee JI, lavarone A, et al. 2018. Hypermutagenesis in untreated adult gliomas due to inherited mismatch mutations. Int J Cancer 144: 3023-3030. doi:10.1002/ijc.32054 
Saunders CT, Wong WS, Swamy S, Becq J, Murray LJ, Cheetham RK. 2012. Strelka: accurate somatic smallvariant calling from sequenced tumor-normal sample pairs. Bioinformatics 28: 1811-1817. doi:10.1093/ bioinformatics/bts271

Stupp R, Hegi ME, Mason WP, van den Bent MJ, Taphoorn MJ, Janzer RC, Ludwin SK, Allgeier A, Fisher B, Belanger K, et al. 2009. Effects of radiotherapy with concomitant and adjuvant temozolomide versus radiotherapy alone on survival in glioblastoma in a randomised phase III study: 5-year analysis of the EORTC$\mathrm{NCIC}$ trial. Lancet Oncol 10: 459-466. doi:10.1016/S1470-2045(09)70025-7

Van der Auwera GA, Carneiro MO, Hartl C, Poplin R, Del Angel G, Levy-Moonshine A, Jordan T, Shakir K Roazen D, Thibault J, et al. 2013. From FastQ data to high confidence variant calls: the Genome Analysis Toolkit best practices pipeline. Curr Protoc Bioinformatics 43: 11.10.11-11.10.33. doi:10.1002/ 0471250953.bi1110s43

Vogelstein B, Papadopoulos N, Velculescu VE, Zhou S, Diaz LA, Kinzler KW. 2013. Cancer genome landscapes. Science 339: 1546. doi:10.1126/science.1235122

Von Hoff DD, Stephenson JJ Jr, Rosen P, Loesch DM, Borad MJ, Anthony S, Jameson G, Brown S, Cantafio N, Richards DA, et al. 2010. Pilot study using molecular profiling of patients' tumors to find potential targets and select treatments for their refractory cancers. J Clin Oncol 28: 4877-4883. doi:10.1200/jco.2009.26 .5983

Wang K, Li M, Hakonarson H. 2010. ANNOVAR: functional annotation of genetic variants from high-throughput sequencing data. Nucleic Acids Res 38: e164. doi:10.1093/nar/gkq603

Wang Q, Hu B, Hu X, Kim H, Squatrito M, Scarpace L, deCarvalho AC, Lyu S, Li P, Li Y, et al. 2017. Tumor evolution of glioma-intrinsic gene expression subtypes associates with immunological changes in the microenvironment. Cancer Cell 32: 42-56.e46. doi:10.1016/j.ccell.2017.06.003 


\section{COLD SPRING HARBOR Molecular Case Studies}

\section{A case study of a long-term glioblastoma survivor with unmethylated MGMT and hypermutated genotype}

Toni Rose Jue, Lauren R. Olafson, Anna H. Siddell, et al.

Cold Spring Harb Mol Case Stud 2019, 5: a003251

Access the most recent version at doi:10.1101/mcs.a003251
Supplementary http://molecularcasestudies.cshlp.org/content/suppl/2019/05/29/mcs.a003251.D Material C1
References This article cites 48 articles, 11 of which can be accessed free at: http://molecularcasestudies.cshlp.org/content/5/3/a003251.full.html\#ref-list-1
License This article is distributed under the terms of the Creative Commons Attribution-NonCommercial License, which permits reuse and redistribution, except for commercial purposes, provided that the original author and source are credited.
Email Alerting Receive free email alerts when new articles cite this article - sign up in the box at the Service top right corner of the article or click here.

